\title{
Sequentially reinforced additive coating for transparent and durable superhydrophobic glass
}

Shuangjie Zhao ${ }^{\mathrm{a}, 1}$, Juan Zhao ${ }^{\mathrm{b}, 1}$, Min Wen ${ }^{\mathrm{a}, 1}$, Ming Yao ${ }^{\mathrm{a}}$, Fengjue Wang ${ }^{\mathrm{a}}$, Fuzhi

Huang $^{\mathrm{a}}$, Qi Zhang ${ }^{\mathrm{c}}$, Yi-Bing Cheng ${ }^{\mathrm{a}, \mathrm{d}}$ and Jie Zhong ${ }^{\mathrm{a}^{*}}$

${ }^{a}$ State Key Laboratory of Advanced Technology for Materials Synthesis and Processing, Wuhan University of Technology, Wuhan 430070, P. R. China

${ }^{b}$ School of Automobile Engineering, Wuhan University of Technology, Wuhan 430070, P. R. China

${ }^{c}$ School of Aerospace, Transport and Manufacturing, Cranfield University, Cranfield, Bedfordshire, MK43 0AL, United Kingdom.

${ }^{d}$ Department of Materials Science and Engineering, Monash University, VIC 3800, Australia

*Corresponding Email: jie.zhong@whut.edu.cn

${ }^{1}$ Those authors contribute equally to this work 


\section{ABSTRACT}

Now that there are various routes to prepare superhydrophobic surfaces for self-cleaning, anti-icing, liquid collecting and etc., attentions are moving towards low-cost upscaling route and increasing the reliability for actual applications. However, the required micro-nano structures for the superhydrophobicity are light scattering and very vulnerable to abrasion, which intrinsically conflicts with the transparency and durability, existing as the major barrier for commercialization of superhydrophobic glass. In this study, we present a novel sequentially reinforced additive coating (SRAC) process to realize robust and transparent micro-nano structured film with tough intergranular sintering. A benign aqueous-based ink with poly(furfuryl alcohol) (PFA) and silica species are carefully designed and sprayed on glass to enable self-phase separation and morphology construction. The coatings reach the static contact angle (SCA) for water over $166^{\circ}$ and withstand a $6 \mathrm{H}$ pencil scratching, the cross-cut test and sands abrasion. Moreover, we also perform 90 days out-door performance test and the glass maintains superhydrophobicity with SCA of $154^{\circ}$. These results provide a low cost waterborne ink formula, and the high throughput and up-scalable SRAC process could be a convenient technology for fabrication large area robust superhydrophobic coatings.

Keywords: superhydrophobic glass; furfuryl alcohol; sequential reinforcement; durable; transparent 


\section{INTRODUCTION}

Self-cleanable surfaces that enable anti-external contamination from either nature or humanity activities are extremely appealing for the upcoming intelligent buildings or vehicles, which could save billions of dollars in personnel and accessories costs to maintain the aesthetic transparency for windows. ${ }^{1-5}$ One of the most desirable measure to realize this function is to construct the superhydrophobic surface coating that water droplets in-combine with the dirt could roll off. ${ }^{6-7}$ Resembling to the lotus leaf, ${ }^{8}$ which is admired as the model for biomimic synthesizing of superhydrophobic coating, fabrications of multi-leveled micro-nano structure are required..$^{9-12}$ The roughness (on the micro and nano-scales) enables the trapping of air under water droplets, thereby contributing to repelling of water droplet to form a static contact angle (SAC) over $150^{\circ}$. However, as-regarding to superhydrophobic glass, the required rough structures, intrinsically conflicts with the transparency and durability, because the textured part is light scattering and vulnerable to abrasion. This challenge inhibits obtaining high performance superhydrophobic glass and causes substantial barrier for commercial applications. ${ }^{13-14}$

Current reported methods for fabrication roughness to realize superhydrophobicity can be generally classified into two categories, the "top-down" and the "bottom-up" measures. ${ }^{15}$ The "top-down" techniques including laser ablation, ${ }^{16-17}$ etching, ${ }^{18-19}$ galvanic corrosion, ${ }^{20}$ photolithography ${ }^{21-22}$ and etc. ${ }^{23-24}$ construct rough structures as the integrated parts of the substrate, contributing to the relatively high mechanical durability. While processability of these measures are typically dependent on 
substrates materials, and their relatively low throughput is not accommodated to the square kilo-meters scale applications cost-effectively. ${ }^{25-27}$ On the other hand, the "bottom-up", 28-30 processes utilized pre-prepared nano/micro building blocks ${ }^{31-32}$ with various shapes ${ }^{33-36}$ such as rods, spheres, core-shells, random agglomerates and etc. to pack and establish the textured surfaces, showing considerable adaptability to the current low-cost industrial painting technologies. The "bottom-up" processing, in contrast to the "top-down" methods, requires additional design to construct tough connections between the substrate and the topographic structure to maintain the water repelling ability. Forming hybrid organic-inorganic bonded composites, ${ }^{30,33,37-41}$ such as polydimethylsiloxane (PDMS), epoxy-siloxane, thiol-ene resins, polymethyl methacrylate (PMMA), fluorinated-decyl polyhedral oligomeric silsequioxane (FD-POSS) and fluorinated alkyl silane (FAS), have emerged as effective measures to enhance durability for various surface coatings. However, organic reinforcement films are required to increase the bonding strength between the particles and substrate. Moreover, because of the low crystallinity, multi-components complex layer and varied refractive indexes, those coatings inevitably result in a problem of blocking of light. Transparency is normally sacrificed for toughness and superhydrophobicity in organic-inorganic coating.

Thus, a simple, economical and effective way to realize a large-scale production of superhydrophobic glass is crucial for commercial interest. We notice that 3-D printing (additive manufacturing) technology using discrete particles as the building blocks have been successfully applied to fabricate ceramics parts with various shapes and 
microstructures. ${ }^{42-44}$ Most importantly, the inter-particles bonding can be enhanced after heat processing and able to endure extensive mechanical frication, tensile and

pressure. ${ }^{45-47}$ In this report, we present a novel route to enhance mechanical strength of the textured film by mimic the additive manufacturing of ceramics, i.e. a sequentially reinforced additive coating (SRAC) process. Different from the additive manufactured parts with computer aided design macrostructures, the textured film was directly constructed in the additive coating of SRAC process with carful design of precursor. We realized a durable and transparent textured layer for superhydrophobic glass with the high toughness up to $6 \mathrm{H}$ and sustainable to $\sim 500 \mathrm{~g}$ sand abrasion at height of $20 \mathrm{~cm}$. Moreover, we performed a 90 days out-door performance test. The glass maintained superhydrophobic properties with contact angle of $154^{\circ}$. Moreover, this textured structure can be deposited on the glass by spraying the waterborne ink directly, which can be convenient for potential large-scale environmentally friendly and cost-effective application.

\section{Experimental section}

\section{Materials}

Tetraethyl orthosilicate (TEOS), concentrated sulfuric acid, hydrogen peroxide $(30 \%$ AR), ethanol (99.7\%), ammonia solution (25-28\%), furfuryl alcohol (FA, 98.5\%), polyvinylpyrrolidone (PVP) were obtained from Sinopharm Chemical Reagent Co. Ltd. Polydimethylsiloxane (PDMS) terminated by $\mathrm{OH}$ group were purchased from Aladdin Co. All of the mentioned reagents were used as received. 


\section{Silica Sol}

$10 \mathrm{ml}$ deionized water was added into $12 \mathrm{ml}$ ethanol. Then $2 \mathrm{ml}$ TEOS was quickly dripped in the mixture of ethanol and water. This solution would be stirred at $600 \mathrm{rpm}$ for $15 \mathrm{~s}$. After that, $0.2 \mathrm{ml}$ ammonia solution was dripped into the mixture under continuous string. Then the stirring rate would be raised to $900 \mathrm{rpm}$. The final mixture is kept at $25^{\circ} \mathrm{C}$ for $24 \mathrm{~h}$.

\section{PFA Ink}

Typically, we added $80 \mathrm{ml}$ deionized water into a flask. Then $2 \mathrm{~g}$ PVP was added into the flask. The mixture should be stirred at $600 \mathrm{rpm}$ until PVP completely dissolved. Then $1 \mathrm{ml}$ FA was added into the flask. 10 copies of this solution should be prepared. The samples were reacted at different times $(6-18 \mathrm{~h})$ at $80{ }^{\circ} \mathrm{C}$ in oil bath to investigate the reaction time. The temperatures are also varied for stirring $\left(60-180{ }^{\circ} \mathrm{C}\right)$ for $12 \mathrm{~h}$ in oil bath. When the heating process started, the stirring speed is raised to $1000 \mathrm{rpm}$. The liquids should be cooled to room temperature when these reactions ended, then they were poured into centrifuge tubes for separation. After 20 min centrifugation at $9000 \mathrm{rpm}$, the brown deposit left at the bottom of each tube was washed with the deionized water. Finally, $6 \mathrm{ml}$ ethanol was poured into each tube. It would be through ultrasonic treatment (200W 30min) until the brown dope was dispersed.

\section{Superhydrophobic Films}

The obtained $\mathrm{SiO}_{2}$ sol was mixed with different amounts of PFA. Typically, $1 \mathrm{ml}$ silica sol was mixed with $0.5-2.5 \mathrm{ml}$ PFA. Soda-lime-silica glass was used as substrates and was washed with ultrapure water and ethanol. The cleaned substrates 
were heated to $220^{\circ} \mathrm{C}$, and then mixture of $\mathrm{SiO}_{2}$ and PFA was sprayed onto glass. The spraying rate was $4 \mathrm{ml} / \mathrm{min}$. The SRCA glass substrates were annealed in air at $520^{\circ} \mathrm{C}$ for $1 \mathrm{~h}$ and cooled naturally. The coated substrates after sintering were soaked into Piranha solution (volume ratio of concentrated sulfuric acid to hydrogen peroxide $(30 \%$ AR) is $7: 3$ ) for $3 \mathrm{~min}$. After this, the films should be washed by deionized water and dried. Then an ethanol solution containing $1 \mathrm{wt} \%$ PDMS would be dipped onto the films to cover them, dried naturally in air for $30 \mathrm{~s}$ and then placed on a heating platform at $320{ }^{\circ} \mathrm{C}$ for $4 \mathrm{~min}$. Among the obtained films, the samples that were produced by the PFA reacting at $150{ }^{\circ} \mathrm{C}$ for $12 \mathrm{~h}$ were denoted as $\mathrm{F} 1, \mathrm{~F} 2, \mathrm{~F} 3, \mathrm{~F} 4$ and F5, according to the different amounts of PFA $(0.5 \mathrm{ml}, 1 \mathrm{ml}, 1.5 \mathrm{ml}, 2 \mathrm{ml}$ and $2.5 \mathrm{ml})$.

\section{Tempered Superhydrophobic Glass}

$0.002 \mathrm{~g} \mathrm{NaCl}$ was added in the each F1, F2, F3, F4 and F5 inks. They were sprayed on auto-glass at $220^{\circ} \mathrm{C}$. After it, the temperature was raised up to $670{ }^{\circ} \mathrm{C}$ in $40 \mathrm{~min}$ and kept for $100 \mathrm{~s}$ in air. Then the glass with the coating was quenched and cooled naturally in the air. $0.25 \mathrm{ml}$ hexane solution containing $1 \mathrm{wt} \%$ PDMS would be sprayed on it for $30 \mathrm{~s}$. Then the glass was heated at $280{ }^{\circ} \mathrm{C}$ for 10 minutes. The obtained films were denoted as S1, S2, S3, S4 and S5.

\section{Characterizations}

The morphologies of the particles and the films were examined via transmission electron microscopy (TEM, JEM-2100F STEM/ EDS), scanning electron microscopy (SEM, ULTRA PLUS-43-13) and optical microscopy (Olympus BX51). The contact angles of different films were measured using a contact angle tester (Theta Lite, 
Biolin Scientific) in the sessile mode at room temperature. The stylus profiler was the DektakXT model. The transmittance of the films was measured on an ultraviolet and visible spectrophotometer (UV-vis, PerkinElmer 750). The FT-IR was Nexus model from Therno Nicolet. The TG/DTA test was measured on integrated thermal analyzer from Netzsch STA449F3. The pencil-scratching test was carried out using a pencil hardness tester according to State Standard Testing Method (GB/T6739-1996), equivalent of the American Society for Testing and Materials (ASTM D3363). A pencil was held firmly against the film at a $45^{\circ}$ and pushed forward by the tester at a speed of $0.5 \mathrm{~mm} \cdot \mathrm{s}^{-1}$. Cross-cut tape adhesion test is according to the American Society for Testing and Materials (ASTM D3359), equivalent to the ISO 2409. The particle sizes and Zeta potentials results were obtained by Malvin analyzer (Mastersizer 3000).

\section{RESULTS AND DISCUSSIONS}

To obtain the textured film, we conceive an organic-inorganic hybrid waterborne ink composed of silica nanocrystal and poly(furfuryl alcohol). Furfuryl alcohol is a type low cost renewable monomer and can be polymerized into PFA at various conditions such as acid and heat inclusion. Moreover, by employing the low-cost and water-ethanol ink materials, it promises environment-friendly manufacturing. After a simple sequentially reinforced additive coating process the superhydrophobic glass was obtained, shown in Figure 1. It contains 4 major steps of ink preparation, deposition, annealing and low surface energy treatment to achieve required properties. 
The silica and PFA precursors were first prepared separately and then mixed as the hybrid ink (see details in the experimental session). The organic-inorganic species were additively coated on the glass substrate and self-assembled into required textures. The sacrificial pattern was constructed by crossed linked PFA, which induced self-phase separation from matrix and is removed by annealing in atmosphere. We prepared a $10 * 10 \mathrm{~cm}^{2}$ sized superhydrophobic glass after low surface energy treatment of the annealed glass with good transparency (Figure 1).

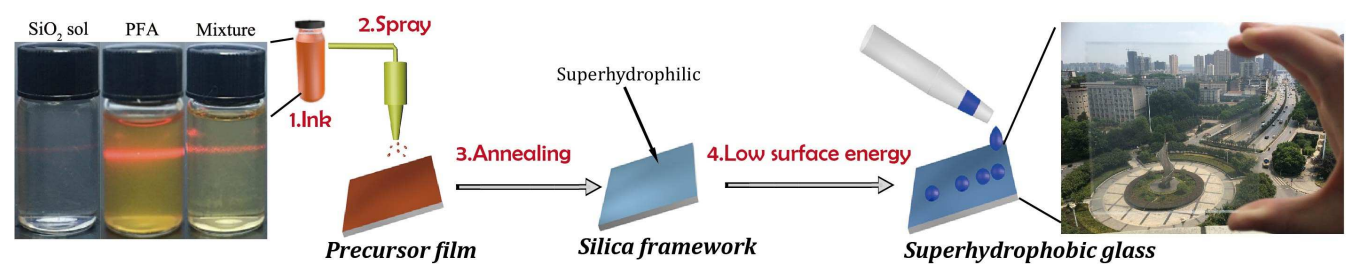

Figure 1. Schematic diagram of the sequentially reinforced additive coating (SRAC). Inserted: (left) The silica sol, poly(furfuryl alcohol) and the mixture ink demonstrating the Tyndall effects; (right) The transparent superhydrophobic glass prepared from the SRAC process.

The key issue during up-mentioned process is how to enable superhydrophobic repelling and also maintain high transparency and robustness. As can be noted in Figure 1, silica, PFA and the hybrid ink demonstrated obvious Tyndall effects, indicating colloidal dispersion. This is confirmed by the dynamic laser scattering (DLS) test. The particle sizes are around $10 \mathrm{~nm}$ and $300 \mathrm{~nm}$ for silica and PFA, respectively. However, the particles size in the mixture increased, with an average increasing to $\sim 800 \mathrm{~nm}$. This resulted from the interaction between PFA and silica, forming larger agglomerates. The peaked Zeta potential of the mixture $(-6.28 \mathrm{mV})$ is just located between silica $(-8.15 \mathrm{mV})$ and PFA $(-3.02 \mathrm{mV})$ indicating certain 
connection happen in two species. However, no new bonds for silica and PFA mixture were suggested in the FTIR curves (Figure 2c), indicating possible hydrogen bonding and static electrical attraction for PFA and silica particles. Figure $2 \mathrm{~d}$ shows the TEM image of PFA ink. The diameters of PFA spheres varied from tens of nanometers to micrometers with bottleneck connections. These perfect PFA spheres were disappeared in mixture (Figure 2e) and covered with the nano sized silica sol (inserted in Figure 2e). These observations suggest PFA and silica have been homogenously mixed and forming nano-micro scale multi-level architectures. This is a benefit to form required porous channel for superhydrophobicity after PFA being removed.

(a)

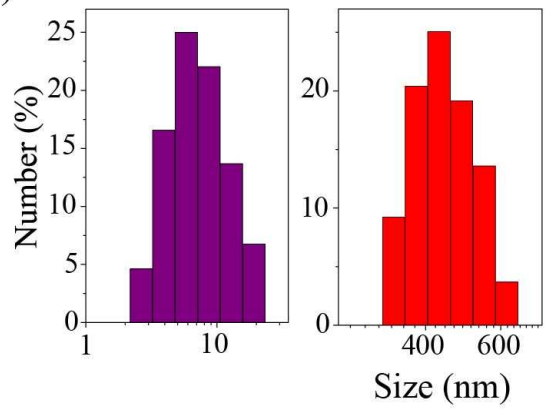

(b)
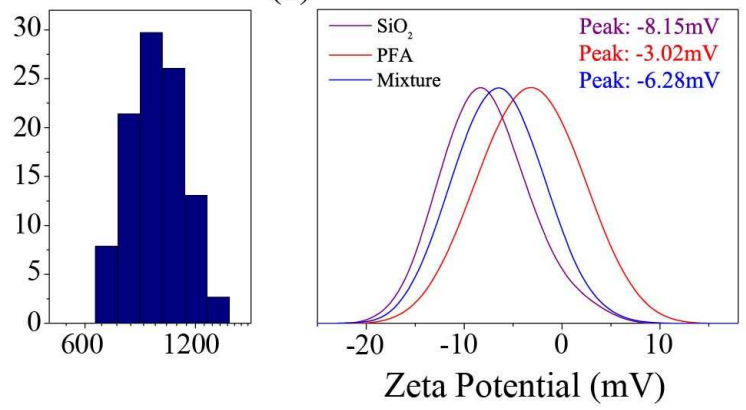

(c)

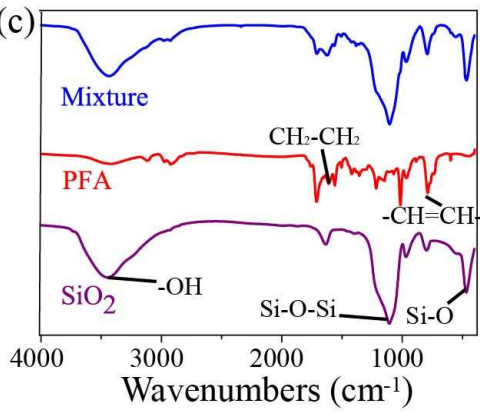

(d)

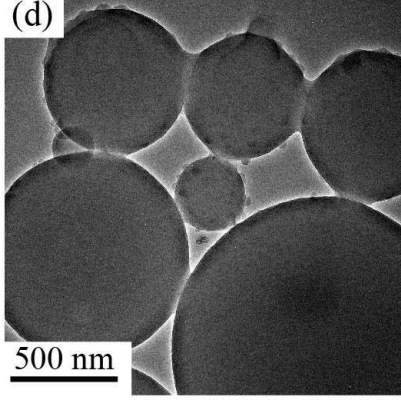

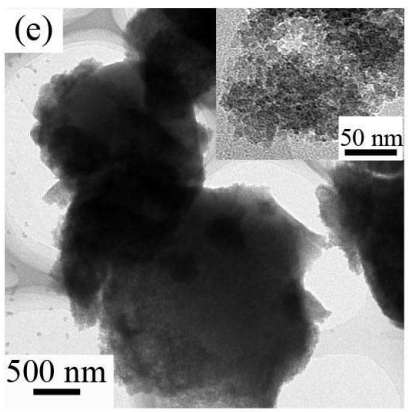

Figure 2. (a) The DLS results of $\mathrm{SiO}_{2}$ sol, PFA and the mixture ink. The vertical axis represents the proportions of each particle size. The Zeta potential patterns (b) and FTIR curves (c) of $\mathrm{SiO}_{2}$ sol, PFA and the mixture. The TEM images of PFA particles (d) and the mixture (e). The image of silica species is inserted. 
As a kind of polymerizable monomer, with different synthetic conditions, such as the temperature and aging time, FA will transform into PFA with varied molecular weight in the solution. The polymerization process would affect the hydrophobicity and cause varied self-phase separation ${ }^{48}$ and morphology construction, and thusly the superhydrophobicity and robustness of the SRCA films. The silica is mixed and bonded with PFA molecules in the nanometer scales. This phase separation is basically due to the PFA agglomeration and silica grain growth during solvent evaporation and temperature elevation. The morphology of porous architecture of silica film indicates the removing of PFA phases which are separated from the homogeneous silica-PFA solution precursor. All of those variables are crucial effects to the SRAC process for the micro-nano structure construction, and thus to the hydrophobicity of the final products. Figure 3 shows the hydrophobicity performances of samples with PFA prepared at different aging temperature and time. The samples are named as PFA-X, where $\mathrm{X}$ stands for the volume ratio of PFA to silica sol. For example, PFA-1.5 means that the proportion of PFA to $\mathrm{SiO}_{2}$ is 1.5 . With the increasing of aging time (Figure $3 \mathrm{a}$ ) at $80{ }^{\circ} \mathrm{C}$, the samples demonstrated similar trend of rapid increasing hydrophobicity and reached a plateau at around $12 \mathrm{~h}$. All samples aged over $12 \mathrm{~h}$ are superhydrophobic except PFA-0.5. The average static contact angles increase with more PFA inclusion and reach a highest value of $170^{\circ}$ for PFA-2.5 aging for $18 \mathrm{~h}$. The similar result of enhancing the hydrophobicity with PFA concentration was observed for the samples prepared at different temperatures. The optimized aging temperature is $150{ }^{\circ} \mathrm{C}$ when the majority samples are 
superhydrophobic. The corresponding photographs of the static contact angles for water droplets are inserted in Figure 3.

(a)

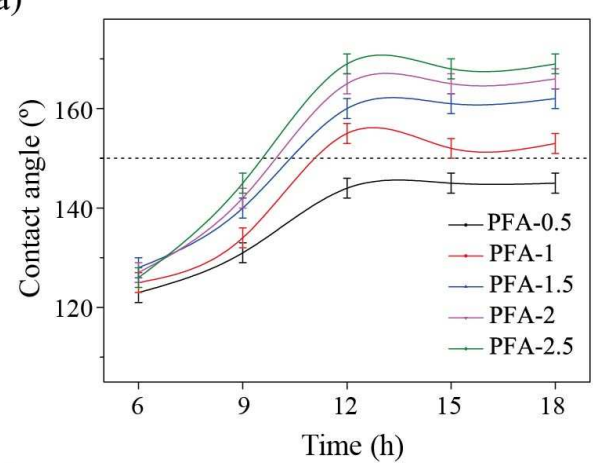

(c)

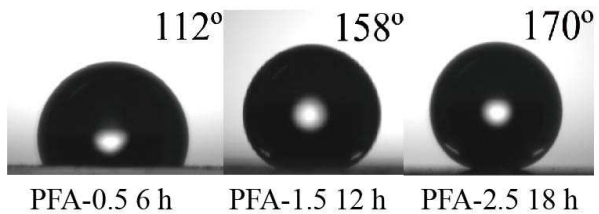

(b)

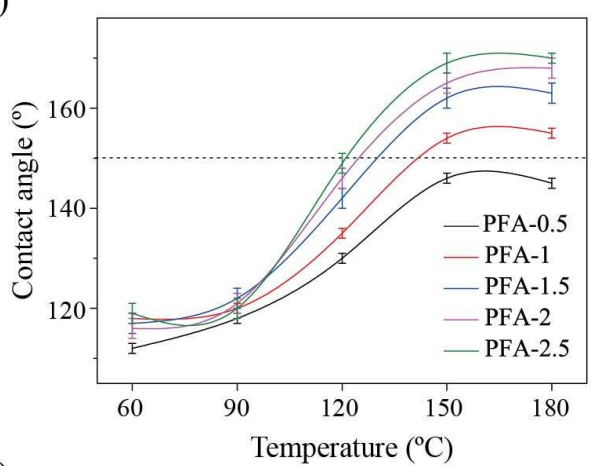

(d)

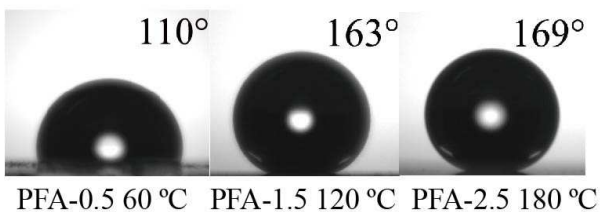

Figure 3. The effect of the polymerization conditions of PFA (aging time and temperature) on the hydrophobic performances of SRAC processed films. The PFA-0.5, PFA-1, PFA-1.5, PFA-2 and PFA-2.5 represent the different volume ratios of PFA/silica of $0.5,1,1.5,2$ and 2.5 , respectively. The SCA results of at (a) the different aging time and (b) the different aging temperatures of PFA. Optical images of the contact angle of water droplets (c) varied aging time and (d) varied aging temperature.

We fixed the heating temperature at $150{ }^{\circ} \mathrm{C}$ and the aging time for $12 \mathrm{~h}$, and the samples were named as $\mathrm{F} 1$ to $\mathrm{F} 5$ with the changing of $\mathrm{PFA} / \mathrm{SiO}_{2}$ volume ratio from 0.5 to 2.5 as shown in Figure $4 \mathrm{a}$. With the increasing $\mathrm{PFA} / \mathrm{SiO}_{2}$ volume ratio, the hydrophobicity of the SRAC processed film was promoted, similar to Figure 3. The water contact angles of the samples appear almost linearly increasing from below $150^{\circ}$ of F1 to $\sim 170^{\circ}$ of F5. However, from the UV-vis tests, the average transmittance shows opposite trend that decreases from over $90 \%$ to $70 \%$ (Figure $4 \mathrm{~b}$ ). This phenomenon is ascribed to the micro-nano structure construction with more PFA involved. The rougher surface was obtained, which induces more light-scattering. We 
also confirmed this proposal by increasing SRAC volume of precursor. As shown in Figure $4 \mathrm{c}$, when SRAC volume of the ink was increased (comparing to F3), the dramatic decreasing of transparency (at $600 \mathrm{~nm}$ wavelength) was observed with slightly enhanced SCA. It seems that the SCA reaches the highest plateau around the value of $170^{\circ}$ as the continuous increasing of ink volume ratio to $\mathrm{F} 3$, whereas the transmittance continues dropping rapidly as the increasing of the ink volume. In the premise of high transmittance and contact angle, 1 to 1.25 times of $\mathrm{F} 3$ is a proper range, with the transparency over $80 \%$ and the SCA over $160^{\circ}$.

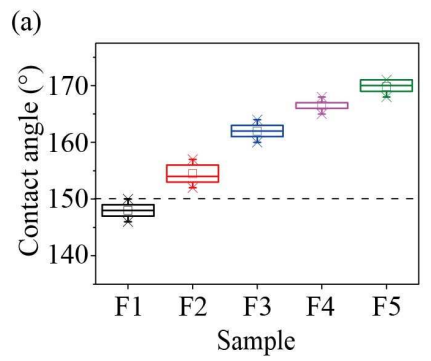

(b)

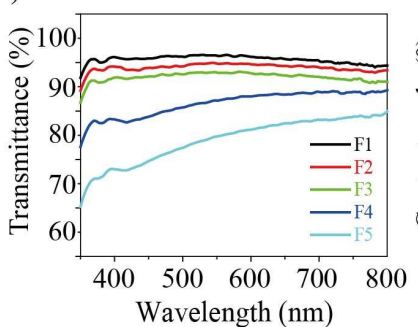

(c)

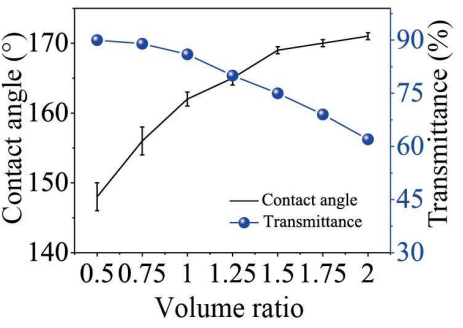

(d)

F2

F3

F4

F5

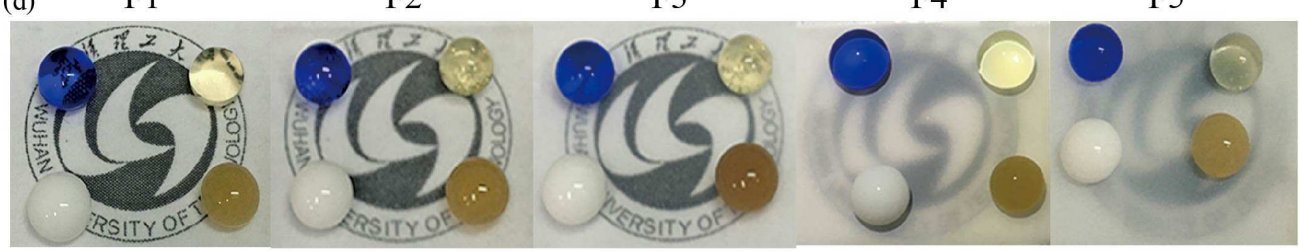

Figure 4. (a) The SAC box plots and (b) the transmittance of samples with varied PFA/silica volume ratio in the mixture ink. The samples with volume ratios of $0.5,1,1.5,2$ to 2.5 are named as F1, F2, F3, F4 and F5, respectively. (c) The effect of SRCA ink volume (ratio to F3) on hydrophobic performances and transmitances of samples. The transmittances are obtained at $600 \mathrm{~nm}$ wavelength. (d) The photographs of F1,F2,F3,F4 and F5 with different water solutions droplets such as fountain pen ink (blue), milk (white), coffee (brown) and juice (yellow).

The photographs of superhydrophobic glass F1 to F5, demonstrating in Figure 4d, suggest well performed superrepelling to the common water solutions such as fountain pen ink, milk, coffee and juice. The tranparency of the samples decreased 
from F1 to F5, and the printed logo of Wuhan University of Technology became vaguing as the increasing of PFA containts.

The SEM morphologies of as-derived films show porous structures (Figure 5a of F1, F2, F3, F4 and F5) with the voids derived from carbon removal. According to the Cassie model, the formula can be rewritten as follow:

$$
\cos \theta^{\mathrm{app}}=r f \cos \theta-1+f .
$$

$\theta^{\text {app }}$ is the apparent contact angle on the surface, $f$ is the fraction of the projected area of the solid surface in contact with the liquid, $r$ is the roughness of the solid surface that is in contact with water and $\theta$ is the equilibrium contact angle on a smooth surface. A small value of $f$ is the key to the construction of superhydrophobic films with continuous pore structure. As shown in Figure 5a, with the amount of PFA increasing, there are more and more pores on the surface and the silica structure is relatively slimmer. Such phenomena make more air remain, then lead to the significant decrease of projected area of the solid surface in contact with the liquid. So, the contact angle was increasing from F1 to F5.

The separated pores gradually evolve to interconnected channels as the increasing of PFA from F1 to F3. And this structure became finer when PFA was further increased for F5. This unique channeled structure facilitates air flowing when liquid droplets cover the pore and also promotes the sintering to obtain reinforced silica framework. The micrographs of the samples after scratching with $4 \mathrm{H}$ pencils are demonstrated in Figure 5b, suggesting obviously enhanced mechanical strength of F3 with no observable scratch mark even at $4 \mathrm{H}$ scratching (the scratching by different are shown 
in Figure S1). Moreover, the superhydrophobic glass prepared from SRAC is also sustainable to $500 \mathrm{~g}$ sand abrasion ${ }^{49-51}$ with the CA maintains over $150^{\circ}$ (Figure S2 and S3). The hardness and CA for samples is shown in Figure 5c. Although the hardness varied with composition, the stylus profiling suggested only F1 film was fully stripped by pencil scratching. As showing in Figure 5a, the special structure, coffee ring, makes main contribution to the high hardness. More silica species are concentrated on the edges of the coffee rings, which make the walls of rings much tougher. Moreover, the higher thickness of the walls could protect the relatively brittle structure inside the rings from abrasion. The thickness of the SRAC films increased with the addition of PFA from $\sim 2 \mu \mathrm{m}$ to $\sim 7 \mu \mathrm{m}$, indicating smaller pores and thinner framework walls were formed at high PFA inclusion in accord with the SEM observation. 
(a)
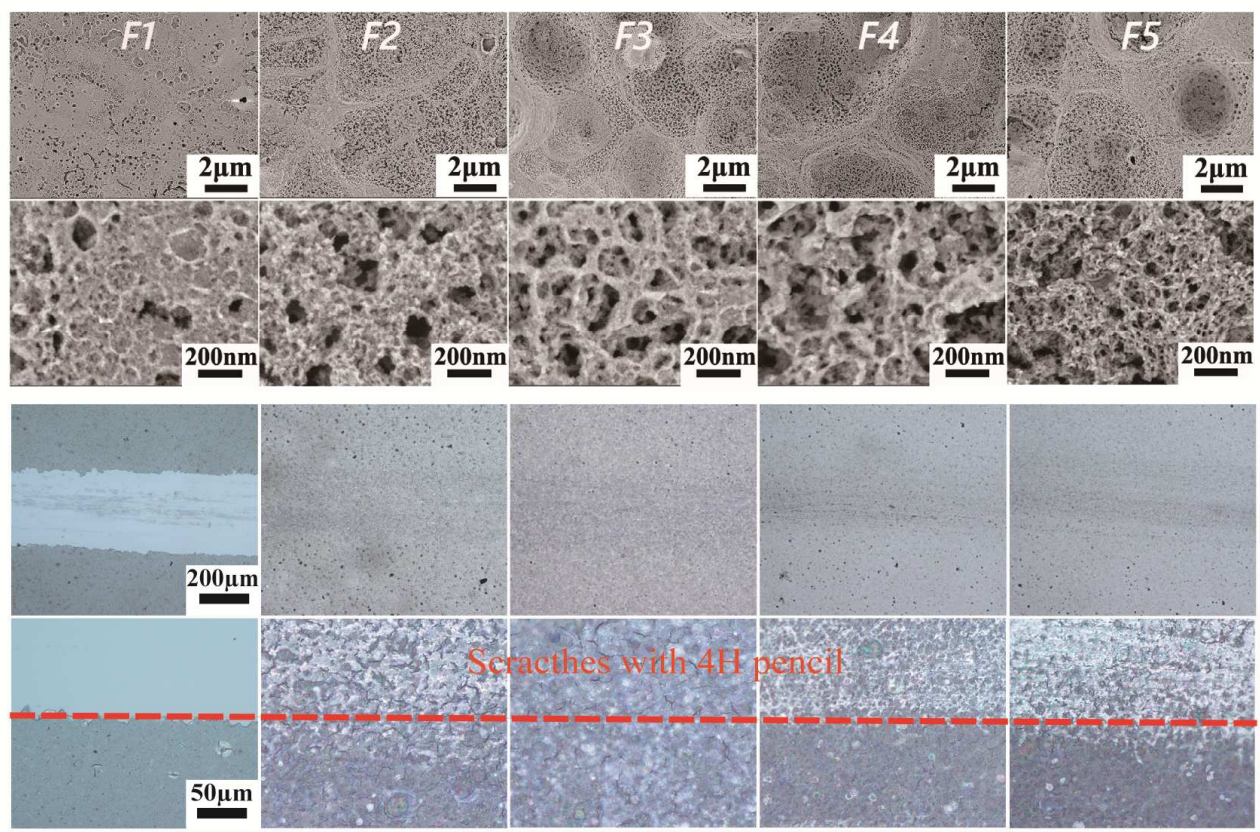

(c)

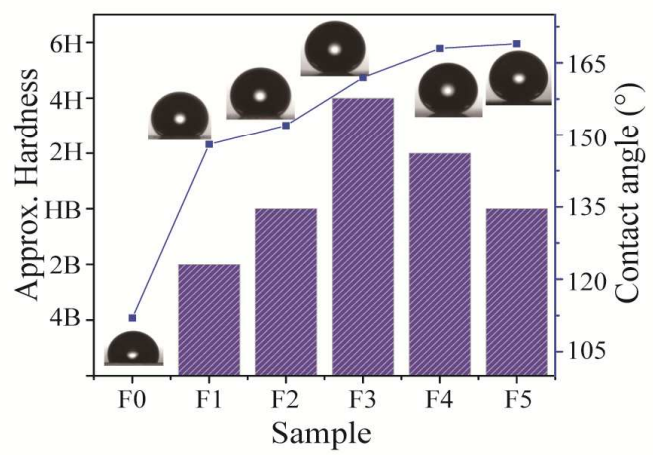

(d)

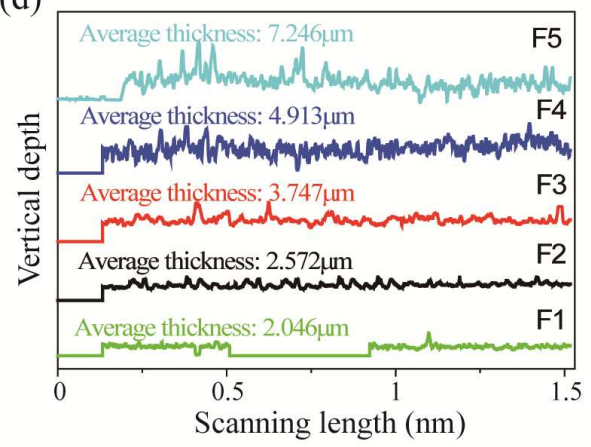

Figure 5. (a) SEM morphologies of samples F1, F2, F3, F4 and F5. The 10 micrometer sized textures were obtained from spray coating. (b) The optical microscope images of $4 \mathrm{H}$ pencil scratches with suggesting varied the hardness of F1 to F5. (c) The approximate hardness and contact angles relations and (d) stylus profiles of the $4 \mathrm{H}$ pencil scratches of F1, F2, F3, F4 and F5.

No separated regions are stripped off for F3 film in a cross-cut tape adhesion test

(Figure S4), indicating very tough bonding between the structured film and the bottom glass substrate. We further elevate the annealing temperature of $\mathrm{F} 3$ to $670{ }^{\circ} \mathrm{C}$ (renamed as S3), similar to the glass tempering process. The received superhydrophobic surface exhibits even higher hardness up to $6 \mathrm{H}$, shown in Table 1. The improvement of mechanical strength is due to $\mathrm{Na}$ ion and elevated temperature, 
which promoted the sintering of silica framework. The detailed results such as pencil scratching, contact angels, stylus profile, transmittance and etc. are demonstrated in Figure S5, S6, S7 and S8 in support information. The resistance to abrasion has been substantially improved with slightly decreasing of CA, indicating high potential of SRCA process to the glass production. A ratio of 1 to 1.25 times of S3 ink volume is the proper to obtain samples with transparency over $75 \%$ and the SCA over $160^{\circ}$ (Figure S9).

In the $3 \mathrm{D}$ printing, means are required to support the free-standing, disconnected features and keep all the features of the part in place during the fabrication process. We consider that the interaction of PFA and silica presents the key function of supporting. The PFA polymers have branched molecular structure allowed them soften and solidify organic-inorganic film. ${ }^{52-54}$ This is also supported by the formation of inconsecutive film of powder like surface from silica ink alone, in contrast to the tough hybrid coating from hybrid ink (Figure S10). The thermal gravity analysis (TG) and differentiate thermal analysis (DTA) results suggest the silica precursor presents only a massive weight loss of $\sim 20 \%$ at around $100-150{ }^{\circ} \mathrm{C}$, which can be ascribed to the removing of residue solvent and hydroxyl condensation. No obvious weight lost was observed for silica in the following heat treatment up to $700{ }^{\circ} \mathrm{C}$. The decomposing for PFA happened around $325^{\circ} \mathrm{C}$, and the further elevation of temperature to $\sim 475^{\circ} \mathrm{C}$ fully removed the PFA species into carbon oxides.

As mentioned above, when the PFA-silica hybrid lost $\mathrm{H}, \mathrm{O}$ and partial organic groups at elevated temperatures, the residue carbon framework could still provide rigid 
supporting to silica nano parts. This could be observed in the TEM images of the particles scraped from the SRAC film Figure $6 \mathrm{~b}$. The denoted red cycles are correlated to the vacuum of removed PFA/carbon spheres, with the pore size around $500 \mathrm{~nm}$ to $30 \mathrm{~nm}$, forming multi-level channeled structure. This facilitates inter-granular sintering and forming high strength nano-structured film.

It can be concluded that PFA plays an important role in the construction of the micro-nanostructure in the process of coating and annealing. Due to its thermosetting property, the PFA skeletons can remain and support the silica framework until the decomposition of carbon. This effect can balance the contraction of silica in the sintering process, and then benefit the silica framework sintering. When the temperature reaches $400{ }^{\circ} \mathrm{C}$, there are only carbon skeleton and silica left and the silica has started sintering. This could be partially supported by the tiny weight loss and exothermal peak at TG and DTA curves, respectively, indicating for further removing of organic species attached to the surface of silica particles. The EDS mapping images of films treated at different temperatures (Figure S11) also reveal the carbon removing process. We thus proposed a sequential reinforcement as following (Figure 6c): 1) The PFA-silica agglomerates are spraying and attached on the glass substrate; 2) PFA is acting as the binder for the powders during the additive deposition and form interconnected network to solidify the precursor film. 3) The hybrid film is strengthened by PFA network at the temperature up to $300{ }^{\circ} \mathrm{C}$, and further supported by PFA-derived carbon network with temperature up to $450{ }^{\circ} \mathrm{C}$; 4) The nano sized silica species start to sinter and be transformed into grain-bounded 
silica film with required texture when PFA is removed. Please note that, reinforcement steps are sequentially taken the major responsibility to support the consecutive film, while the boundary temperatures for those mechanisms may not be defined exactly.

(a)

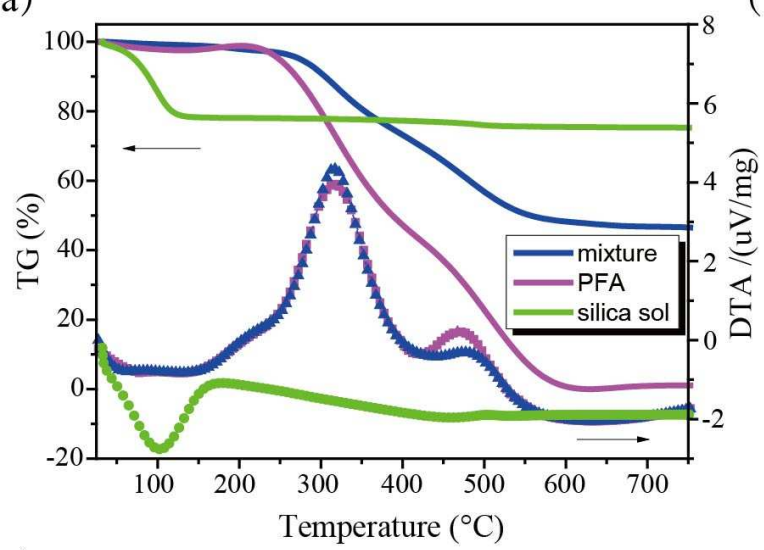

(b)

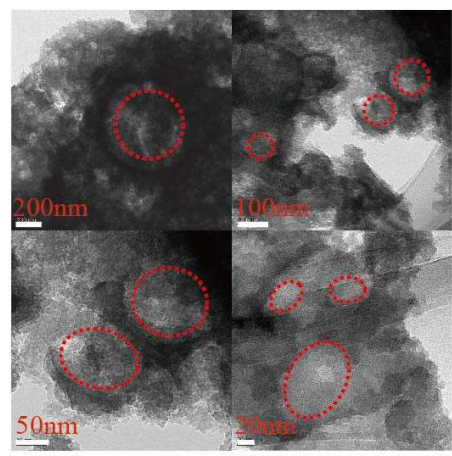

(c)

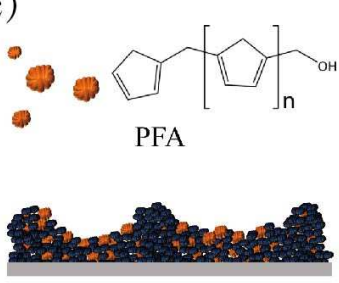

PFA-silica composite

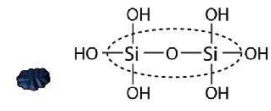

silica

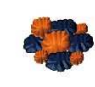

Bonded silica-PFA $300-400^{\circ} \mathrm{C}$

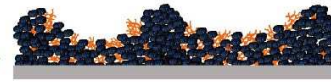

Silica-carbon composite

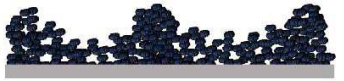

Sintered silica framework

Figure 6. (a) The TG/DTA curves of silica sol, PFA and mixture. (b) The TEM images of the mixture when it has been heated to $400^{\circ} \mathrm{C}$. (c) The Schematic diagram of the strengthen process. 
Table 1. The selected properties of superhydrophobic glass samples of PFA-0 F3, F5 and S3.

\begin{tabular}{lllll}
\hline Samples & Contact & Roll-off & Pencil & Transmittance \\
\hline PFA-0 & angle $\left(^{\circ}\right)$ & angle $\left(^{\circ}\right)$ & hardness & $(\mathbf{6 0 0 n m})$ \\
F3 (PFA-1.5, $\left.500{ }^{\circ} \mathrm{C}\right)$ & $100 \pm 1$ & $>90$ & $<10 \mathrm{~B}$ & - \\
F5 (PFA-2.5, $\left.500{ }^{\circ} \mathrm{C}\right)$ & $162 \pm 2$ & $3-5$ & $4 \mathrm{H}$ & $\sim 87 \%$ \\
S3 (PFA-1.5, $\left.0.004 \mathrm{~g} \mathrm{NaCl}, 670^{\circ} \mathrm{C}\right)$ & $162 \pm 2$ & $3-5$ & $6 \mathrm{H}$ & $86 \%$ \\
\hline
\end{tabular}

For the aim of application, we carried out an outdoor test demonstration. The sample was placed in open air at a $45^{\circ}$ angle with superhydrophobic surface outwards in Wuhan, China for a continued period of 3 months. The recorded CA results are shown in Figure 7 along with the weather information. The temperature variations of this period are shown in Figure S12. According to China's National Ambient Air Standard GB3095-2012 and Technical Regulation on Ambient Air Quality Index HJ633-2012, AQI (air quality index) contains a rating on six substances, including PM2.5, PM10, $\mathrm{SO}_{2}, \mathrm{NO}_{2}, \mathrm{O}_{3}$ and $\mathrm{CO}$. The highest one among the values is published, which are majorly on PM2.5 in cities. When AQI value over 100 is considered as pollution and the density of PM2.5 reaches $75 \mu \mathrm{g} / \mathrm{cm}^{3}$. It seems that the high AQI weather will decrease the $\mathrm{CA}$ of the glass, but the rain flush could create remedy for the hydrophobicity to some extent, demonstrating self-cleaning effect. Moreover, ultraviolet light also causes damage to the performance probably due to the degradation of organic coating. The CA decreased to $\sim 154^{\circ}$ after 3 months continuous exposing outdoor weather, maintaining superhydrophobicity. This result suggested high application potential for SRCA film with further modifications. 


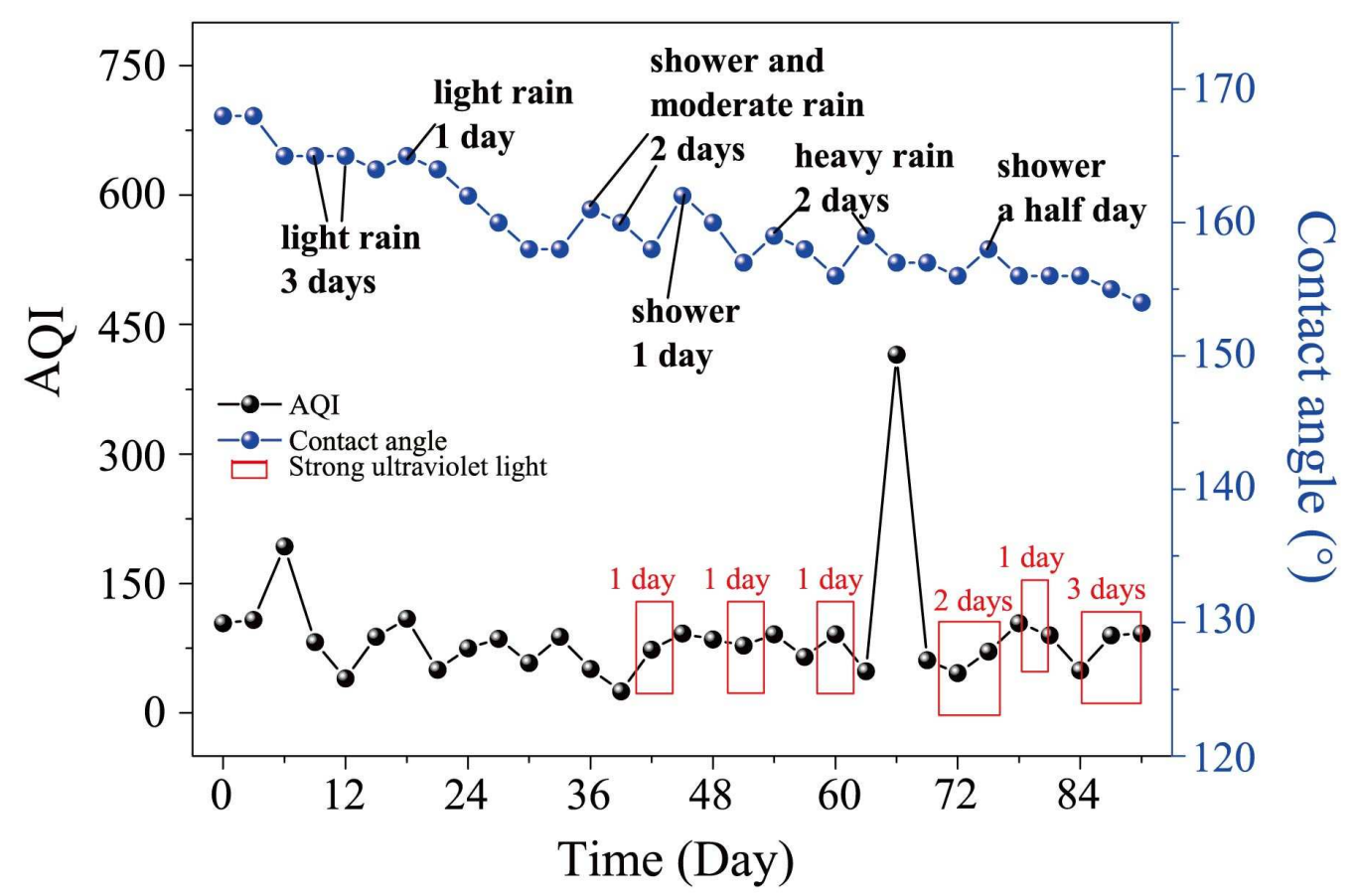

Figure 7. 90-day outdoor examination of F3 sample in Wuhan. Strong ultraviolet light weather means the ultraviolet radiation reaching the ground is $15-30 \mathrm{~W} / \mathrm{m}^{2}$.

\section{CONCLUSION}

In summary, we present a facile sequentially reinforced additive coating (SRAC) process to fabricate transparent robust superhydrophobic glass. The aqueous ink with poly furfuryl-alcohol (PFA) and silica species are carefully designed, and spray deposited on glass. Through delicate ink design and heating process, we enabled self-phase separation and sequentially reinforcement for the silica to achieve robust and transparent superhydrophobic films. The PFA aging conditions and PFA/ratio exhibit varied morphology and mechanical strength. The robust micro-nano structured film with tough intergranular sintering, which can withstand $4 \mathrm{H}$ pencil test, cross-cut test and sand abrasion. The SCA for water is as high as $164^{\circ}$ and the transmittance 
can be as high as $87 \%$. This SRAC method can be combined with glass-tempered process. We realized a durable and transparent textured layer for superhydrophobic glass with the high hardness up to $6 \mathrm{H}$. Moreover, we performed 90 days out-door performance test. The glass maintained superhydrophobicity with the contact angle of $154^{\circ}$. Thus, this convenient waterborne SRAC method is promising for up-scaling fabrication of superhydrophobic glass environmentally friendly and cost-effectively.

\section{Associated Content}

Supporting Information. Brief statement in nonsentence format listing the contents of the material supplied as Supporting Information.

\section{Acknowledgement}

This work is financially supported by National Natural Science Foundation of China (NSFC 51402115), Hubei Provincial Natural Science Foundation of China (2016CFB464), the Technological Innovation Key Project of Hubei Province (2016AAA041). QZ would like to thank the support of "Hubei one-hundred Plan". JZ thanks the support the "Chutian Scholar Program" of Hubei Province, China. 


\section{REFERENCES}

(1) Tian, X.; Verho, T.; Ras, R. H. A. Moving superhydrophobic surfaces toward real-world applications. Science 2016, 352, 142-143.

(2) Wen, L.; Tian, Y.; Jiang, L. Bioinspired super-wettability from fundamental research to practical applications. Angewandte Chemie 2015, 54, 3387-99.

(3) Tian, Y.; Su, B.; Jiang, L. Interfacial material system exhibiting superwettability. Advanced materials 2014, 26, 6872-97.

(4) Yao, X.; Song, Y.; Jiang, L. Applications of bio-inspired special wettable surfaces. Advanced materials 2011, 23, 719-34.

(5) Su, B.; Tian, Y.; Jiang, L. Bioinspired Interfaces with Superwettability: From Materials to Chemistry. Journal of the American Chemical Society 2016, 138, 1727-48.

(6) Gao, L.; McCarthy, T. J. The "Lotus Effect” Explained: Two Reasons Why Two Length Scales of Topography Are Important. Langmuir 2006, 22, 2966-2967.

(7) Barthlott, W.; Schimmel, T.; Wiersch, S.; Koch, K.; Brede, M.; Barczewski, M.; Walheim, S.; Weis, A.; Kaltenmaier, A.; Leder, A.; Bohn, H. F. The Salvinia Paradox: Superhydrophobic Surfaces with Hydrophilic Pins for Air Retention Under Water. Advanced materials 2010, 22, 2325-2328.

(8) Darmanin, T.; Guittard, F. Superhydrophobic and superoleophobic properties in nature. Materials Today 2015, 18, 273-285.

(9) Hwang, H. S.; Kim, N. H.; Lee, S. G.; Lee, D. Y.; Cho, K.; Park, I. Facile fabrication of transparent superhydrophobic surfaces by spray deposition. ACS applied materials \& interfaces 2011, 3, 2179-83.

(10) Cassie, A. B. D.; Baxter, S. Wettability of porous surfaces. Transactions of the Faraday Society 1944, 40, 546-551.

(11) Kreder, M. J.; Alvarenga, J.; Kim, P.; Aizenberg, J. Design of anti-icing surfaces: smooth, textured or slippery? Nature Reviews Materials 2016, 1.

(12) Wang, B.; Liang, W.; Guo, Z.; Liu, W. Biomimetic super-lyophobic and super-lyophilic materials applied for oil/water separation: a new strategy beyond nature. Chemical Society reviews 2015, 44, 336-61.

(13) Li, J.; Jing, Z.; Zha, F.; Yang, Y.; Wang, Q.; Lei, Z. Facile spray-coating process for the fabrication of tunable adhesive superhydrophobic surfaces with heterogeneous chemical compositions used for selective transportation of 
microdroplets with different volumes. ACS applied materials \& interfaces 2014, 6, 8868-77.

(14) Darmanin, T.; Guittard, F. Recent advances in the potential applications of bioinspired superhydrophobic materials. J. Mater. Chem. A 2014, 2, 16319-16359.

(15) Chen, L.; Guo, Z.; Liu, W. Outmatching superhydrophobicity: bio-inspired re-entrant curvature for mighty superamphiphobicity in air. Journal of Materials Chemistry A 2017, 5, 14480-14507.

(16) Jiang, H.-B.; Zhang, Y.-L.; Han, D.-D.; Xia, H.; Feng, J.; Chen, Q.-D.; Hong, Z.-R.; Sun, H.-B. Bioinspired Fabrication of Superhydrophobic Graphene Films by Two-Beam Laser Interference. Advanced Functional Materials 2014, 24, 4595-4602.

(17) Vorobyev, A. Y.; Guo, C. Direct femtosecond laser surface nano/microstructuring and its applications. Laser \& Photonics Reviews 2013, 7, 385-407.

(18) Xiu, Y.; Zhu, L.; Hess, D. W.; Wong, C. P. Hierarchical Silicon Etched Structures for Controlled Hydrophobicity/Superhydrophobicity. Nano Letters 2007, 7, 3388-3393.

(19) Khedir, K. R.; Saifaldeen, Z. S.; Demirkan, T. M.; Al-Hilo, A. A.; Brozak, M. P.; Karabacak, T. Robust Superamphiphobic Nanoscale Copper Sheet Surfaces Produced by a Simple and Environmentally Friendly Technique. Advanced Engineering Materials 2015, 17, 982-989.

(20) Xufeng, W.; Gaoquan, S. Fabrication of a lotus-like micro-nanoscale binary structured surface and wettability modulation from superhydrophilic to superhydrophobic. Nanotechnology 2005, 16, 2056.

(21) Kang, H.; Heo, Y. J.; Kim, D. J.; Kim, J. H.; Jeon, T. Y.; Cho, S.; So, H. M.; Chang, W. S.; Kim, S. H. Droplet-Guiding Superhydrophobic Arrays of Plasmonic Microposts for Molecular Concentration and Detection. ACS applied materials \& interfaces 2017, 9, 37201-37209.

(22) Wang, S.; Liu, M.; Feng, Y.; Bu, Y.; Huynh, S. H.; Ng, T. W.; Gu, F.; Yu, A.; Jiang, X. Bioinspired hierarchical copper oxide surfaces for rapid dropwise condensation. J. Mater. Chem. A 2017, 5, 21422-21428. 
(23) Deng, X.; Mammen, L.; Butt, H. J.; Vollmer, D. Candle soot as a template for a transparent robust superamphiphobic coating. Science 2012, 335, 67-70.

(24) Liu, T. L.; Kim, C.-J. C. Turning a surface superrepellent even to completely wetting liquids. Science 2014, 346, 1096.

(25) Si, Y.; Guo, Z. Superhydrophobic nanocoatings: from materials to fabrications and to applications. Nanoscale 2015, 7, 5922-5946.

(26) Li, X. M.; Reinhoudt, D.; Crego-Calama, M. What do we need for a superhydrophobic surface? A review on the recent progress in the preparation of superhydrophobic surfaces. Chemical Society reviews 2007, 36, 1350-68.

(27) Zhang, X.; Shi, F.; Niu, J.; Jiang, Y.; Wang, Z. Superhydrophobic surfaces: from structural control to functional application. J. Mater. Chem. 2008, 18, 621-633.

(28) Wang, W.; Lockwood, K.; Boyd, L. M.; Davidson, M. D.; Movafaghi, S.; Vahabi, H.; Khetani, S. R.; Kota, A. K. Superhydrophobic Coatings with Edible Materials. ACS applied materials \& interfaces 2016, 8, 18664-8.

(29) Zhu, Z.; Bai, Y.; Zhang, T.; Liu, Z.; Long, X.; Wei, Z.; Wang, Z.; Zhang, L.; Wang, J.; Yan, F.; Yang, S. High-performance hole-extraction layer of sol-gel-processed $\mathrm{NiO}$ nanocrystals for inverted planar perovskite solar cells. Angew Chem Int Ed Engl 2014, 53, 12571-5.

(30) Zhou, H.; Wang, H.; Niu, H.; Gestos, A.; Lin, T. Robust, Self-Healing Superamphiphobic Fabrics Prepared by Two-Step Coating of Fluoro-Containing Polymer, Fluoroalkyl Silane, and Modified Silica Nanoparticles. Advanced Functional Materials 2013, 23, 1664-1670.

(31) Zhang, L. D.; Liu, W. L.; Xu, W. H.; Yao, J. S.; Zhao, L.; Wang, X. Q.; Wu, Y. Z. Synthesis and characterization of superhydrophobic and superparamagnetic film based on maghemite-polystyrene composite nanoparticles. Applied Surface Science 2012, 259, 719-725.

(32) Lu, Y.; Sathasivam, S.; Song, J.; Crick, C. R.; Carmalt, C. J.; Parkin, I. P. Robust self-cleaning surfaces that function when exposed to either air or oil. Science 2015, 347, 1132.

(33) Xue, C.-H.; Zhang, Z.-D.; Zhang, J.; Jia, S.-T. Lasting and self-healing superhydrophobic surfaces by coating of polystyrene/SiO2nanoparticles and polydimethylsiloxane. Journal of Materials Chemistry A 2014, 2, 15001. 
(34) Xu, Q. F.; Wang, J. N.; Smith, I. H.; Sanderson, K. D. Superhydrophobic and transparent coatings based on removable polymeric spheres. J. Mater. Chem. $2009,19,655-660$.

(35) Wen, M.; Zhong, J.; Zhao, S.; Bu, T.; Guo, L.; Ku, Z.; Peng, Y.; Huang, F.; Cheng, Y.-B.; Zhang, Q. Robust transparent superamphiphobic coatings on non-fabric flat substrates with inorganic adhesive titania bonded silica. J. Mater. Chem. A 2017, $5,8352-8359$

(36) Zhang, G.; Lin, S.; Wyman, I.; Zou, H.; Hu, J.; Liu, G.; Wang, J.; Li, F.; Liu, F.; Hu, M. Robust superamphiphobic coatings based on silica particles bearing bifunctional random copolymers. ACS applied materials \& interfaces 2013, 5, 13466-77.

(37) Zhou, H.; Wang, H.; Niu, H.; Gestos, A.; Wang, X.; Lin, T. Fluoroalkyl silane modified silicone rubber/nanoparticle composite: a super durable, robust superhydrophobic fabric coating. Advanced materials 2012, 24, 2409-12.

(38) Wang, S.; Li, Y.; Fei, X.; Sun, M.; Zhang, C.; Li, Y.; Yang, Q.; Hong, X. Preparation of a durable superhydrophobic membrane by electrospinning poly (vinylidene fluoride) (PVDF) mixed with epoxy-siloxane modified $\mathrm{SiO} 2$ nanoparticles: a possible route to superhydrophobic surfaces with low water sliding angle and high water contact angle. J Colloid Interface Sci 2011, 359, 380-8.

(39) Yilgor, I.; Bilgin, S.; Isik, M.; Yilgor, E. Facile preparation of superhydrophobic polymer surfaces. Polymer 2012, 53, 1180-1188.

(40) Xiong, L.; Kendrick, L. L.; Heusser, H.; Webb, J. C.; Sparks, B. J.; Goetz, J. T.; Guo, W.; Stafford, C. M.; Blanton, M. D.; Nazarenko, S.; Patton, D. L. Spray-deposition and photopolymerization of organic-inorganic thiol-ene resins for fabrication of superamphiphobic surfaces. ACS applied materials \& interfaces 2014, 6, 10763-74.

(41) Zhou, H.; Wang, H.; Niu, H.; Zhao, Y.; Xu, Z.; Lin, T. A Waterborne Coating System for Preparing Robust, Self-healing, Superamphiphobic Surfaces. Advanced Functional Materials 2017, 27, 1604261.

(42) Eckel, Z. C.; Zhou, C.; Martin, J. H.; Jacobsen, A. J.; Carter, W. B.; Schaedler, T. A. Additive manufacturing of polymer-derived ceramics. Science 2016, 351, 58. 
(43) Gross, B. C.; Erkal, J. L.; Lockwood, S. Y.; Chen, C.; Spence, D. M. Evaluation of 3D printing and its potential impact on biotechnology and the chemical sciences. Analytical chemistry 2014, 86, 3240-53.

(44) Wu, C.; Fan, W.; Zhou, Y.; Luo, Y.; Gelinsky, M.; Chang, J.; Xiao, Y. 3D-printing of highly uniform CaSiO3 ceramic scaffolds: preparation, characterization and in vivo osteogenesis. Journal of Materials Chemistry 2012, 22, 12288.

(45) Crossland, E. J.; Noel, N.; Sivaram, V.; Leijtens, T.; Alexander-Webber, J. A.; Snaith, H. J. Mesoporous TiO2 single crystals delivering enhanced mobility and optoelectronic device performance. Nature 2013, 495, 215-9.

(46) Suryanarayana, C.; Al-Aqeeli, N. Mechanically alloyed nanocomposites. Progress in Materials Science 2013, 58, 383-502.

(47) Gu, M.; Li, Y.; Li, X.; Hu, S.; Zhang, X.; Xu, W.; Thevuthasan, S.; Baer, D. R.; Zhang, J.-G.; Liu, J.; Wang, C. In Situ TEM Study of Lithiation Behavior of Silicon Nanoparticles Attached to and Embedded in a Carbon Matrix. ACS nano 2012, 6, 8439-8447.

(48) Zhong, J.; Liang, S.; Xu, C.; Wang, H.; Cheng, Y.-B. Meso/micro-porosity and phase separation in TiO2/SiO2/C nanocomposites. Microporous and Mesoporous Materials 2012, 150, 25-31.

(49) Ren, T.; He, J. Substrate-Versatile Approach to Robust Antireflective and Superhydrophobic Coatings with Excellent Self-Cleaning Property in Varied Environments. ACS applied materials \& interfaces 2017, 9, 34367-34376.

(50) Xu, L.; Geng, Z.; He, J.; Zhou, G. Mechanically robust, thermally stable, broadband antireflective, and superhydrophobic thin films on glass substrates. ACS applied materials \& interfaces 2014, 6, 9029-35.

(51) Deng, X.; Mammen, L.; Zhao, Y.; Lellig, P.; Mullen, K.; Li, C.; Butt, H. J.; Vollmer, D. Transparent, thermally stable and mechanically robust superhydrophobic surfaces made from porous silica capsules. Advanced materials 2011, 23, 2962-5. (52) Moazzen, K.; Zohuriaan-Mehr, M. J.; Jahanmardi, R.; Kabiri, K. Toward poly(furfuryl alcohol) applications diversification: Novel self-healing network and toughening epoxy-novolac resin. Journal of Applied Polymer Science 2018, $135,45921$. 
(53) Drisko, G. L.; Zelcer, A.; Wang, X.; Caruso, R. A.; Soler-Illia, G. J. Synthesis and photocatalytic activity of titania monoliths prepared with controlled macro- and mesopore structure. ACS applied materials \& interfaces 2012, 4, 4123-30.

(54) Kherroub, D. E.; Belbachir, M.; Lamouri, S. Synthesis of poly(furfuryl alcohol)/montmorillonite nanocomposites by direct in-situ polymerization. Bulletin of Materials Science 2015, 38, 57-63. 


\section{For Table of Contents Only}

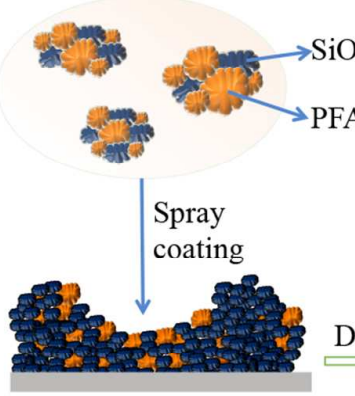

PFA-silica composite

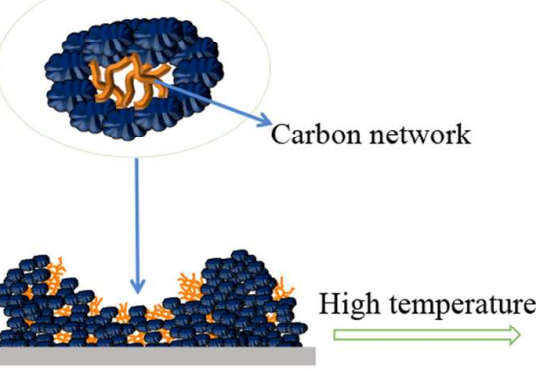

Silica-carbon composite

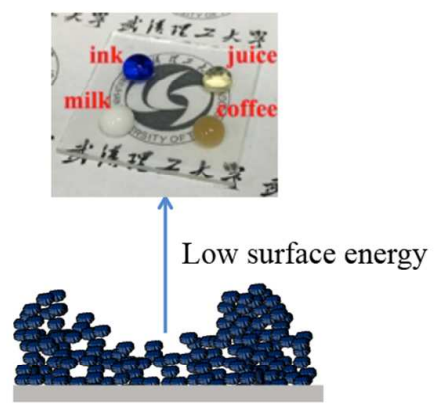

Sintered silica framework 
2018-09-05

\section{Sequentially reinforced additive coating}

for transparent and durable

superhydrophobic glass

\section{Zhao, Shuangjie}

American Chemical Society

Shuangjie Zhao, Juan Zhao, Min Wen, et al., Sequentially reinforced additive coating for

transparent and durable superhydrophobic glass. Langmuir, 2018, Volume 31, Issue 38, pp. 11316-11324

https://doi.org/10.1021/acs.langmuir.8b01960

Downloaded from Cranfield Library Services E-Repository 